\title{
Equitable Admissions in the Time of COVID-19
}

\author{
For this graduate admissions season and beyond, holistic review can help \\ departments look beyond the tip of the iceberg of their applicants.
}

\author{
By Casey Miller and Julie Posselt
}

$\prod$ he COVID-19 pandemic, the Trump administration's restrictions on international students, and a national movement for racial justice are compelling many physics programs in the US to rethink graduate admissions. While many leading programs have begun to make changes in recent years, others now have a new sense of urgency to reexamine their processes. To equitably evaluate applicants and avoid further disadvantaging the students most affected by these many challenges, we advocate for holistic review in graduate admissions-review processes that systematically and thoughtfully take into account a variety of applicant experiences and credentials.

Disruptions due to COVID-19 alone mean that students, through no fault of their own, will submit applications this coming cycle and beyond that look different from those submitted in

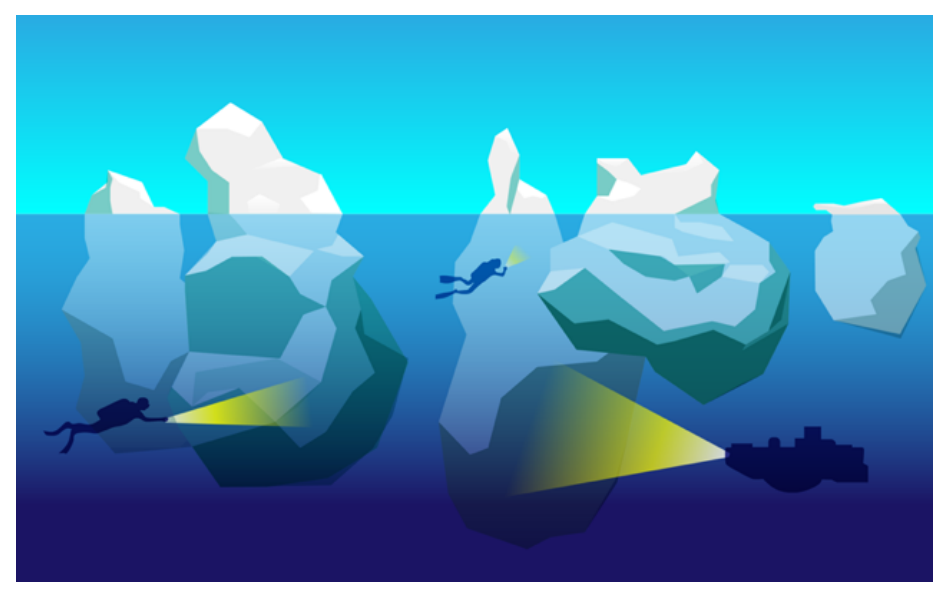

Credit: APS/Alan Stonebraker previous cycles. Institutions across the country are offering students flexibility in how grades are reported; programs will see increased frequency of "pass/fail" or "incomplete" on transcripts. The Graduate Record Examinations (GRE) subject test in physics has been temporarily canceled. Closures and variable levels of campus reopenings have significantly curtailed or canceled research projects that provide substance to applicants' personal statements and recommendation letters. (According to Edmundo Javier Garcia-Solis, a program officer within the Division of Physics of the National Science Foundation (NSF), about 30\% of 2020 Research Experiences for Undergraduates (REU) sites in physics were canceled, while $65 \%$ were run remotely.)

It will be more important than ever that programs account for the variability in student circumstances when assessing their applications. Holistic review can help make such assessments equitable and efficient.

As leads on a team studying equity in graduate admissions, we developed a framework for holistic review that has helped physics Ph.D. programs (among others) make their selection processes nimbler and more equitable. The framework allows departments to address challenges like those presented now. Our work through an NSF INCLUDES Alliance (IGEN) has shown, for example, that admissions committees using our approach (albeit not in isolation) admitted higher fractions of women and minoritized students than their traditional admissions processes historically allowed.

In our estimation, most programs in physics and astronomy can readily move toward holistic review because its elements are 
often already in use, albeit to varying degrees. The approach we recommend is one that is comprehensive, contextualized, and systematic, with equity-mindedness permeating throughout.

To ensure applicant review is both comprehensive and systematic, we suggest that programs should use rubrics to guide their processes. Rubrics help make the process comprehensive by keeping the diverse criteria being considered salient to reviewers. They also provide a structure for meaningfully assessing all applicants on each criterion in a systematic manner. A rubric can include just a handful of high-level dimensions of admissibility, such as academic preparation, scholarly potential, student-program alignment, diversity contributions, and emotional-social competencies. To increase the efficiency of the review process, each of these should have only three or so bins; it is quicker to distinguish "high" from "medium" than "8" from "7." Rubrics are most useful if designed so that the full dynamic range is used, so bins should have clear definitions that can evenly trifurcate the applicants for each dimension. Scholarly potential, for example, might be signaled by (i) a clear passion for research verified by mentors, (ii) multiple years conducting research, (iii) making the most of the research opportunities at their institution, and (iv) notable research products, such as a senior thesis or conference presentation. For an applicant to receive a "high," they might satisfy all four of these, "medium" might be (i) plus any other two, and "low" might be two or fewer of these.

Contextualization means looking below the surface to understand why portions of an application might look the way they do. We need to think about students' records in light of the opportunities and constraints they have faced. Often, the best way to do this is by triangulating information within the application: Is the Grade Point Average (GPA) lower than expected because of a rough semester or two in 2020, or perhaps when the student first entered college, suggesting they may simply have needed time to adjust to the change? Did a family situation or the digital divide (gaps in access to digital tools) prevent the applicant from being able to fully meet conventional expectations? Were the student's research options shuttered because of COVID-19?

Contextualization and equity-mindedness go hand in hand. Among other things, they require awareness that gender, race, socioeconomic status, national origin, and type of undergraduate institution affect how a student has fared during the pandemic. The pandemic has sharpened existing inequalities, and applications will reflect this problem. Equity-mindedness also means being conscious that the review process-and the humans who carry it out-can inadvertently be biased against certain groups, and that the likelihood of this bias is greater when reviewers are themselves facing stressors.

COVID-19 has certainly made clear how situations unrelated to one's potential in physics may affect what committees will see in applications. We can leverage this terrible situation though: Changes catalyzed by the pandemic can improve equity in future admissions cycles and broaden access to physics graduate education. For those of you interested in going a bit deeper, we have provided some guidance for self-reflection to assess whether your program's admissions process is where it needs to be or if it has fallen out of phase with the program's aspirations or competition. Whether you seek to manage admissions this year or make your admissions process more equitable for the long term, holistic review can help.

Correction (22 December 2020): An earlier version implied that the cancellation of the GRE subject test in physics could have been permanent.

Casey Miller: Rochester Institute of Technology, Rochester, NY, USA

Julie Posselt: University of Southern California, Los Angeles, CA, USA 